\title{
MODELING OF LOW-DIMENSIONAL CARBON NANOSTRUCTURES: AN EFFICIENT APPROACH BASED ON CHEMICAL CRITERIA
}

\author{
Francesco Mercuri, Matteo Baldoni, Antonio Sgamellotti \\ ISTM-CNR, INSTM and University of Perugia, via Elce di Sotto 8, 06123 Perugia, Italy \\ merc@thch.unipg.it
}

Recently, the properties of nanostructured carbon materials, like carbon nanotubes (CNTs) and graphene, have been the subject of in-depth investigations in view of their potential use in nanotechnology. Most of the interest concerning nanostructured carbon materials is related to the peculiarities of their electronic structure, which is constituted mainly by a complex network of pi-conjugated bonds. Details of the electronic structure play a crucial role in the application of such materials as nanostructured building-blocks for molecular electronics and in functionalization processes, where the CNTs and graphenes undergo chemical reactions[1]. However, the particular structural and electronic features of nanostructured carbon poses significant problems to computational modeling. Recent work indicated the extension of classical organic chemistry concepts to the case of low-dimensional nanostructured carbon materials as a successful approach to obtain an accurate and consistent description of the electronic structure of the hexagonal carbon atom network[2-4]. In this work we apply state-ofthe-art numerical techniques to investigations on the stability and on electronic and chemical properties of nanotubes and graphenes and related compounds[4-6]. Our approach is based on the definition of suitable models of the system under study starting from chemical considerations. Results indicate unprecedented accuracy in the prediction of properties for a large variety of systems, obtained at a relatively cheap computational cost.

\section{References:}

[1] F. Mercuri, A. Sgamellotti, Inorg. Chim. Acta 360 (2007) 785.

[2] M. Baldoni, A. Sgamellotti, F. Mercuri, Org. Lett. 9 (2007) 4267.

[3] F. Mercuri, Mol. Sim. 34 (2008) 905.

[4] M. Baldoni, A. Sgamellotti, F. Mercuri, Chem. Phys. Lett. 464 (2008) 202.

[5] F. Mercuri, A. Sgamellotti, Phys. Chem. Chem. Phys. 11 (2009) 563.

[6] M. Baldoni, D. Selli, A. Sgamellotti, F. Mercuri, J. Phys. Chem. C 113 (2009) 862. 
Figures:

Oral
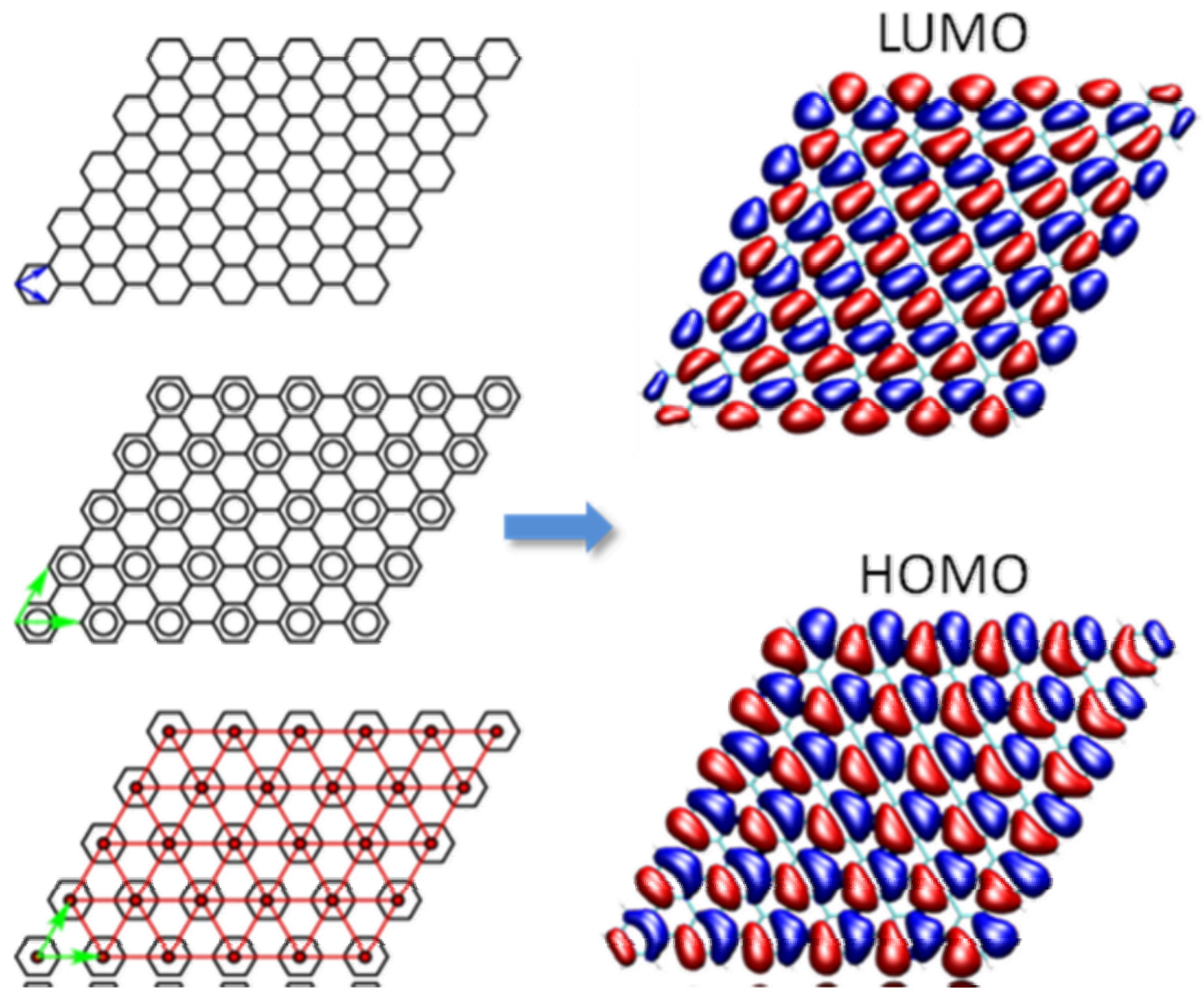